\title{
Confiabilidad de un cuestionario para medir la actividad física y los comportamientos sedentarios en niños desde preescolar hasta cuarto grado de primaria
}

\author{
Diana Marina Camargo, Stefany Santisteban, Erika Paredes, Mary Ann Flórez, Diego Bueno \\ Grupo de Investigación "Movimiento, Armonía y Vida", Escuela de Fisioterapia, Facultad de Salud, \\ Universidad Industrial de Santander, Bucaramanga, Colombia
}

\begin{abstract}
Introducción. Las recomendaciones internacionales sobre actividad física y tiempo dedicado a comportamientos sedentarios en niños a partir de la edad preescolar, plantean la necesidad de disponer de instrumentos de medición con propiedades psicométricas que permitan evaluar la dinámica a nivel de la población y las intervenciones dirigidas a mejorar su salud.

Objetivo. Evaluar la confiabilidad de un cuestionario para medir la actividad física y los comportamientos sedentarios en niños desde preescolar hasta cuarto grado de primaria.

Materiales y métodos. Ciento ocho padres respondieron el cuestionario, el cual incluía preguntas sobre las variables sociodemográficas y las relacionadas con la actividad física, incluido el tiempo de caminata hasta el colegio, los deportes organizados y las actividades de juego; entre los comportamientos sedentarios se incluyeron el transporte motorizado a la escuela, el tiempo de lectura, el transcurrido frente a pantallas y el sueño. Mediante el coeficiente alfa de Cronbach, el coeficiente de correlación intraclase y el método de Bland y Altman, se evaluaron la consistencia interna, la reproducibilidad y los límites de acuerdo, respectivamente.
\end{abstract}

Resultados. La consistencia interna osciló entre 0,59 y 0,64 para la actividad física y entre 0,22 y 0,34 para los comportamientos sedentarios. Los mejores niveles de reproducibilidad se registraron para la caminata (kappa $=0,79)$, el tiempo de viaje a la escuela $(\mathrm{CCl}=0,69)$, el deporte organizado ( $\mathrm{kappa}=0,72$ ), el tiempo dedicado a este $(\mathrm{CCl}=0,76)$, el transporte motorizado al colegio y el tiempo empleado para ello (kappa $=0,82 ; \mathrm{CCl}=0,8$ ), así como para el uso del computador y el tiempo dedicado a esta actividad (kappa $=0,71 ; \mathrm{CCl}=0,59)$. Se registraron niveles de acuerdo de moderados a buenos para el tiempo de lectura, la siesta, los cursos extracurriculares, y el uso de computador y de consolas.

Conclusión. El cuestionario suministró información confiable para la medición de la actividad física y los comportamientos sedentarios en niños menores de 10 años y podría emplearse en otros países latinoamericanos.

Palabras clave: actividad motora, estilo de vida sedentario, cuestionarios, reproducibilidad de resultados, preescolar.

doi: http://dx.doi.org/10.7705/biomedica.v35i3.2502

Reliability of a questionnaire for measuring physical activity and sedentary behavior in children from preschool to fourth grade

Introduction: International recommendations for physical activity and time spent in sedentary behaviors for children in their early years require the availability of measuring instruments with psychometric properties that allow for the assessment of population dynamics and interventions to improve health.

Objective: To evaluate the reliability of a questionnaire to measure physical activity and sedentary behaviors in children from preschool to fourth grade.

Materials and methods: One hundred and eight parents answered the questionnaire. The instrument included socio-demographic variables, as well as those associated with physical activity, including time walking to school, organized sports and playtime activities. Sedentary behaviors included motorized transport to school, reading and "screen time", sleeping and extracurricular courses. Internal consistency, reproducibility and agreement were evaluated using Cronbach's alpha coefficient, the Intraclass Correlation Coefficient (ICC) and the Bland and Altman limits of agreement method, respectively.

\section{Contribución de los autores:}

Diana Marina Camargo: elaboración de la propuesta de investigación, coordinación del desarrollo del trabajo, análisis de los datos y redacción del manuscrito final

Stefany Santisteban, Erika Paredes, Mary Ann Flórez y Diego Bueno: participación en los ajustes de la metodología del trabajo, diseño del cuestionario, ejecución de la prueba piloto y contribución en la elaboración del manuscrito final 
Results: Internal consistency for physical activity ranged from 0.59 to 0.64 , and for sedentary behaviors between 0.22 and 0.34 . The highest reproducibility was found for walking to school and time spent on this (kappa=0.79, ICC 0.69), and organized sports, and time on this activity (kappa=0.72, ICC 0.76). Among sedentary behaviors, motorized transport to school and computer use showed kappas of 0.82 and 0.71 , respectively; additionally, the time spent on these behaviors showed an ICC of 0.8 and 0.59 , respectively. We found limits of agreement between moderate and good for reading time, napping, extracurricular courses, computer and console use.

Conclusion: The questionnaire provided reliable information on the physical activity and sedentary behaviors in children under 10 years of age and could be used in other Latin American countries.

Key words: Motor activity, sedentary lifestyle, questionnaires, reproducibility of results; child, preschool. doi: http://dx.doi.org/10.7705/biomedica.v35i3.2502

La evidencia científica disponible sobre los efectos positivos de la actividad física en la salud y el bienestar de las personas, ha resultado en la elaboración de recomendaciones aplicables a nivel mundial (1). En este contexto, la medición de los niveles de actividad física se ha convertido en una prioridad, pues permite determinar su frecuencia y su distribución en la población, evaluar la eficacia y la efectividad de las intervenciones dirigidas a su fomento, establecer la relación entre la actividad física y diversos indicadores de salud, así como identificar los determinantes de su práctica (2).

Los métodos para la medición de la actividad física se clasifican en objetivos y subjetivos. En el primer grupo se encuentran la observación directa, la técnica de agua doblemente marcada y la calorimetría indirecta. Asimismo, se cuenta con tres tipos de dispositivos, entre los cuales están los monitores de frecuencia cardiaca, los pedómetros y los acelerómetros. En los niños menores de 10 años, los sistemas de observación directa y los acelerómetros son los más utilizados; además, la evaluación de sus propiedades psicométricas ha dado resultados satisfactorios (3-5).

Entre los métodos subjetivos se cuenta con el reporte de los padres o acudientes o profesores, pues el reporte del propio niño, utilizado en los mayores de 10 años, no se recomienda para los más pequeños debido a que su nivel de desarrollo cognitivo les dificulta la comprensión de las preguntas planteadas en los cuestionarios y, además, su capacidad para recordar las

\section{Correspondencia:}

Diana Marina Camargo, Escuela de Fisioterapia, Universidad Industrial de Santander, Carrera 32 № 29-31, Bucaramanga, Santander, Colombia

Teléfono: (576) 634 4000, extensiones 3381 y 3147; fax: (576) 6358582

dcamargo@uis.edu.co

Recibido: 29/07/14; aceptado: 30/03/15 actividades realizadas es muy limitada. Por otro lado, estos métodos ofrecen varias ventajas, como su fácil aplicación, mayor cobertura y bajo costo. Asimismo, permiten evaluar todas las dimensiones de la actividad física y facilitan la recordación del contexto en que se realizó. Cabe señalar que el recuerdo no altera el comportamiento evaluado (3-5).

La medición de la actividad física en los niños pequeños es un reto debido al nivel de desarrollo de sus habilidades motoras y a otros factores biológicos, sociales y del medio ambiente que la condicionan y la hacen muy variable (6). Por otra parte, se manifiesta a través del juego, mecanismo que les permite explorar su medio ambiente, ampliar su lenguaje y estimular su imaginación y su pensamiento creativo. Los niños suelen ser espontáneos y presentan menos interés en una sola actividad, siguen un patrón de estimulación variada, condicionada por el entorno y, probablemente, por la necesidad de dotarse de nuevas experiencias sensoriales y motoras, por lo que no siempre involucra movimientos que resulten en un gasto energético significativo $(7,8)$.

Diversas agencias y organismos internacionales han propuesto algunas recomendaciones generales para las actividades lúdicas de los niños. En primer lugar, deben ser apropiadas para la edad, agradables y seguras, deben desarrollarse en el contexto del hogar, las instituciones de cuidado, la escuela y la comunidad, tanto en recintos abiertos como cerrados, y bajo la supervisión de un adulto o cuidador. Asimismo, el tiempo dedicado a actividades sedentarias se debe limitar, con excepción del tiempo dedicado a dormir. Se recomienda específicamente que los preescolares acumulen al menos 180 minutos diarios de actividad física de cualquier intensidad (8-10), y en lo que respecta a los comportamientos sedentarios, que el tiempo continuo en sedente no exceda una hora. No se recomienda que los menores de dos años pasen tiempo frente a pantallas de televisión, 
computador, juegos electrónicos o similares, y que en los niños de 2 a 4 años de edad, el llamado "tiempo de pantalla" se limite a menos de una hora diaria (11).

En la literatura científica revisada se encontraron algunos cuestionarios diseñados para medir la actividad física y los comportamientos sedentarios en niños menores de 10 años que indagan sobre diferentes constructos y dimensiones de la actividad física, como son el contexto, las preferencias y la atracción por la actividad física en los niños, y algunos, además, sobre la alimentación, factores todos que se asocian a la obesidad y el sobrepeso (12-22).

Los resultados de la medición de las propiedades psicométricas son muy variables, con coeficientes que fluctúan entre puntuaciones bajas y altas, una consistencia interna moderada y niveles de acuerdo entre bajos y moderados, lo que evidencia la variabilidad del comportamiento de los niños y la capacidad de los padres o cuidadores de registrar la información. En cuanto a la consistencia de criterio, al comparar los cuestionarios con acelerómetros uniaxiales que tienen diferentes puntos de corte, se han registrado coeficientes de correlación de Spearman entre bajos y moderados, y una consistencia interna del constructo de tipo factorial aceptable que explica más del $50 \%$ de la varianza (12-22).

Es importante destacar que en la revisión no se encontraron cuestionarios diseñados para medir la actividad física y los comportamientos sedentarios en niños menores de 10 años en el ámbito latinoamericano. Por lo anterior, el objetivo de este estudio fue elaborar un cuestionario confiable en términos de consistencia interna, reproducibilidad y acuerdo, para medir la actividad física y el comportamiento sedentario dirigido a los padres de niños desde preescolar hasta cuarto grado de primaria matriculados en instituciones educativas de un área urbana de Colombia.

\section{Materiales y métodos}

Se hizo una evaluación de pruebas de diagnóstico, lo cual requirió el diseño de un nuevo cuestionario con base en el análisis de cuestionarios similares dirigidos a los padres de niños en el grupo de edad del estudio, disponibles en la literatura científica consultada (12-22); además, se contó con las cifras de población relacionadas con la prevalencia de los comportamientos activos y sedentarios recolectadas en un trabajo anterior (23).

\section{Población de estudio}

La población de estudio estuvo conformada por los padres de niños escolarizados de ambos sexos matriculados en cinco instituciones educativas de Bucaramanga desde preescolar hasta cuarto grado de primaria. Los padres respondieron el cuestionario en dos oportunidades diferentes con un período intermedio de una semana. La información se recolectó en los colegios, a donde los padres fueron citados entre marzo y junio de 2012 para responder el cuestionario.

\section{Variables de estudio}

Las variables incluidas en el cuestionario indagaron, en primer lugar, por las características sociodemográficas de los padres: sexo, edad, escolaridad, estrato socioeconómico y trabajo fuera de casa. Se preguntaba a continuación por las variables relacionadas con los niños: edad, sexo, grado y jornada escolar (mañana o tarde), y hora de entrada y salida del colegio.

El cuestionario indagaba por la actividad física y los comportamientos sedentarios de los niños durante todos los días de la semana anterior por fuera del colegio; se daban, además, dos ejemplos para ilustrar la forma de diligenciar el cuestionario tanto en lo concerniente a la actividad física como al comportamiento sedentario (véase el anexo, disponible en: doi: http://dx.doi.org/10.7705/ biomedica.v35i3.2502).

En el cuestionario, la actividad física se definió como sigue: "Actividades en las que los niños necesitan un mayor esfuerzo, aumentan los latidos del corazón, la respiración y, a veces, sudan, por ejemplo: caminar, correr, jugar con la pelota o practicar algún deporte". Se incluyeron cuatro atributos: el desplazamiento al colegio caminando, el contexto de juego (dentro y fuera de la casa), el deporte organizado, y ocho actividades de juego, para un total de 12 ítems.

Los comportamientos sedentarios se definieron como aquellos que "Requieren mínimo o ningún movimiento y se necesita poco esfuerzo, por ejemplo: ver televisión, leer un libro o usar el computador, entre otros". Se establecieron cinco atributos: el desplazamiento hacia el colegio en vehículo motorizado, el tiempo dedicado a la lectura, las actividades frente a pantallas (televisión, computadores y consolas), el tiempo de sueño (siesta y en la noche), y los cursos extracurriculares de tipo sedentario, para un total de ocho ítems. 
La respuesta para cada ítem se registró en forma dicotómica, sí o no; una respuesta afirmativa implicaba reportar el tiempo dedicado a la actividad en minutos y horas durante cada día de la semana. Con este fin, se establecieron la frecuencia y la duración en minutos de cada tipo de actividad para los cinco días entre semana (suma del tiempo de lunes a viernes), y los dos días de fin de semana (suma del tiempo durante sábado y domingo).

\section{Procedimiento}

Inicialmente, se evaluó la consistencia aparente del cuestionario con la participación de 11 padres de niños entre los 6 y 11 años de edad. La calificación promedio fue de 4,7/5,0 para el diseño y las imágenes, y de 4,9 para el tamaño de la letra y la claridad de las preguntas. Todos los participantes opinaron que las definiciones de actividad física, de comportamiento sedentario y los respectivos ejemplos,fueron claros; el $20 \%$ de los participantes tuvo la percepción de que el cuestionario era largo, el $100 \%$ reportó que el registro del tiempo en minutos para la actividad física y en horas para los comportamientos sedentarios, fue fácil o muy fácil.

\section{Análisis}

El análisis de confiabilidad se hizo por separado para los atributos e ítems de la actividad física y de los comportamientos sedentarios practicados tanto entre semana como en el fin de semana. Se aplicó el coeficiente alfa de Cronbach para evaluar la consistencia interna, el coeficiente kappa de Cohen para evaluar la reproducibilidad de las variables medidas en escala nominal y el coeficiente de correlación intraclase para las variables medidas en escala de razón, con sus respectivos intervalos de confianza del $95 \%\left(\mathrm{IC}_{95 \%}\right)$ (24). La interpretación de la reproducibilidad se ajustó a las recomendaciones de Landis y Koch (25): ligera, de 0,01 a 0,2; aceptable, de 0,21 a 0,40; moderada, de 0,41 a 0,60; sustancial, de 0,61 a 0,80, y casi perfecta, de 0,81 a 1,00. Además, se determinó el nivel de acuerdo aplicando el método de los límites de Bland y Altman (26). El análisis consideró un nivel de significancia de alfa de 0,05 y se desarrolló en el programa Stata 12.1 (27).

El estudio fue aprobado por el Comité de Ética de la Facultad de Salud de la Universidad Industrial de Santander; el consentimiento informado fue firmado por los rectores de las instituciones educativas seleccionadas aleatoriamente para participar en el estudio y por los padres de familia, y se contó con el asentimiento verbal de los niños participantes.

\section{Resultados}

Se distribuyeron 410 cuestionarios en las agendas escolares. En la primera ocasión, el porcentaje de respuesta fue de $28,8 \%(118 / 410)$ y en la segunda, de $91,5 \%$ (108/118). Por consiguiente, se contó con 108 cuestionarios para el análisis. El $82 \%$ de quienes respondieron correspondió al sexo femenino y el $68 \%$ eran madres, con un promedio de edad de $37,8 \pm 8,9$ años y una escolaridad de 13,4 \pm 3,6 años; el $59 \%$ trabajaba fuera de casa, el $76 \%$ tenía empleo de tiempo completo y la mitad de la muestra tenía un nivel socioeconómico medio.

El promedio de la edad de los niños fue de 7,2 \pm 1,7 años, el $50 \%$ correspondía al sexo femenino; la siguiente fue la distribución por grado escolar: $17 \%$ de prejardín, jardín y preescolar, $34 \%$ de los grados $1^{\circ}$ y $2^{\circ}$ de primaria, y el $49 \%$ de los grados $3^{\circ}$ y $4^{\circ}$. El $58,5 \%$ de los niños estudiaba en la jornada de la mañana.

Las frecuencias de cada tipo de comportamiento se presentan en las figuras 1 y 2 . En cuanto a la actividad física, las mayores cifras se registraron para jugar dentro y fuera de la casa, jugar con los amigos y caminar, y en lo concerniente al comportamiento sedentario, ver televisión, leer, ir en vehículo al colegio y usar el computador.

\section{Consistencia interna}

Los resultados del coeficiente alfa de Cronbach fueron de 0,59 en la primera medición y de 0,62 en la segunda para las variables dicotómicas de los ítems de actividad física, y de 0,60 y 0,64 para los registros del tiempo en las dos mediciones, respectivamente. Los comportamientos sedentarios registraron un coeficiente alfa de Cronbach de 0,22 y 0,31 para las dos mediciones nominales y de 0,34 y 0,23 para las variables de tiempo.

\section{Reproducibilidad}

La reproducibilidad de los ítems de actividad física tanto para las variables medidas en escala nominal como para el tiempo computado en minutos osciló entre moderada y sustancial (cuadro 1), con excepción del tiempo en el parque; para los comportamientos sedentarios se registró una reproducibilidad entre moderada y casi perfecta (cuadro 2) para la mayoría de los ítems.

\section{Límites de acuerdo}

El análisis del acuerdo en los minutos diarios de actividad física entre las dos mediciones arrojó promedios de las diferencias cercanos a cero con 


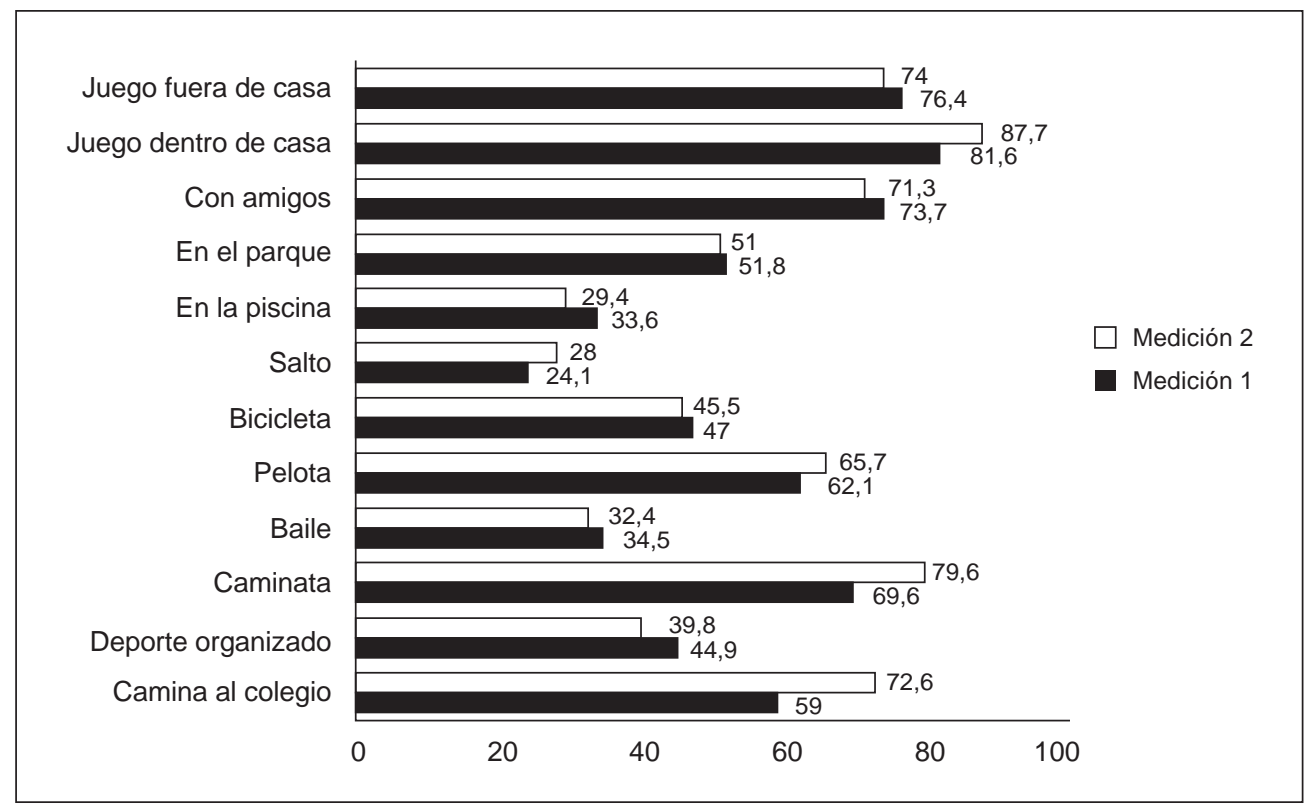

Figura 1. Frecuencia de las actividades de juego y su contexto en las dos mediciones efectuadas

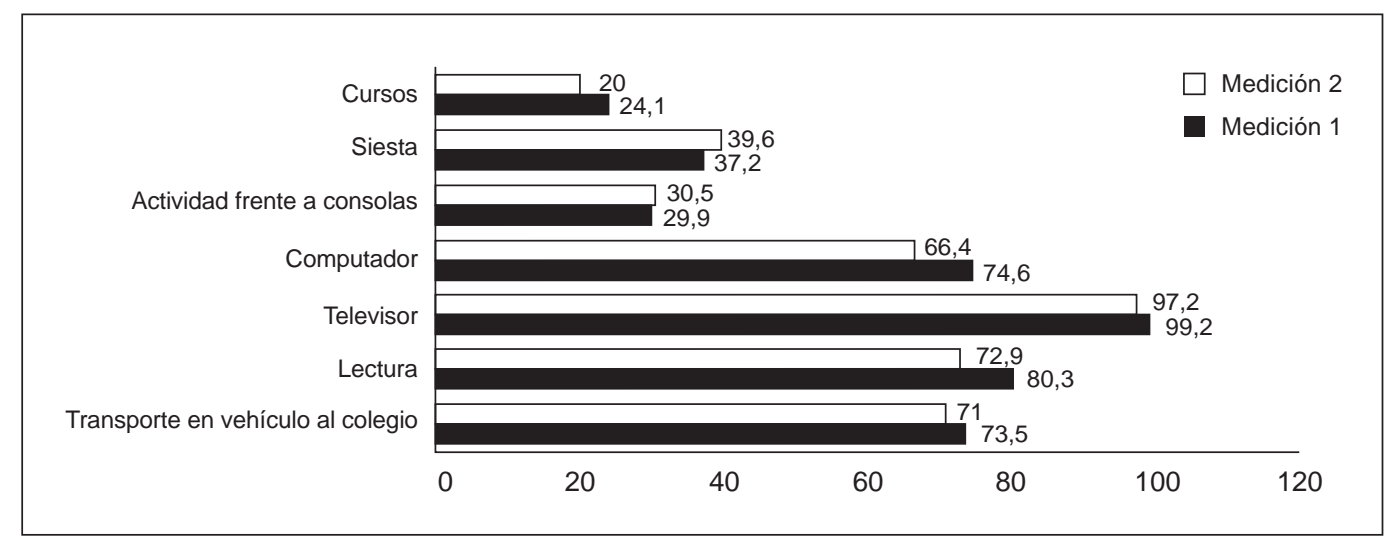

Figura 2. Frecuencia de las actividades sedentarias en las dos mediciones efectuadas

límites próximos a los 30 minutos para la medición total, y una variabilidad mayor al discriminar entre semana y fin de semana (cuadro 3). Se encontraron resultados similares para los comportamientos sedentarios, con excepción de los ítems de sueño en la noche y ver televisión, que registraron la mayor variabilidad (cuadro 4).

\section{Discusión}

La epidemia de sobrepeso y obesidad en los niños, así como la evidencia sobre los efectos positivos de la actividad física en este grupo, otorga relevancia a la medición de los niveles de actividad física entre la población $(5,8,9)$. Por ello, es importante contar con un instrumento cultural y técnicamente adaptado al contexto latinoamericano, en el cual se reconozca el juego como el principal indicador de actividad física y, además, se mida el comportamiento sedentario, ya que ambos hacen parte de las actividades cotidianas de las personas.

La comparación de los hallazgos de este estudio con los de otros cuestionarios, debe partir del constructo establecido en cada instrumento para la definición de la actividad física (tiempo, frecuencia, duración, preferencias, atracción por la actividad), y de lugar (aire libre, en casa, diversos contextos); igualmente, debe analizarse la pertinencia cultural de los ítems incluidos y otros aspectos técnicos del diseño, como el período de medición (día anterior, 
Cuadro 1. Reproducibilidad prueba-reprueba para la práctica de actividad física

\begin{tabular}{|c|c|c|c|c|c|c|}
\hline \multirow[t]{2}{*}{ Atributo } & \multirow[t]{2}{*}{ Ítem } & \multirow[t]{2}{*}{$\mathbf{N}$} & \multirow{2}{*}{$\begin{array}{c}{ }^{\text {a }} \text { Kappa }^{\text {b }}\left(I C_{95 \%}\right) \\
\text { Sí/No }\end{array}$} & \multicolumn{3}{|c|}{${ }^{c} \mathbf{C C l}^{\mathrm{b}} \mathrm{IC}_{95 \%}$} \\
\hline & & & & ${ }^{d}$ ES & ${ }^{\mathrm{e}} \mathrm{FS}$ & Total \\
\hline \multirow{3}{*}{$\begin{array}{l}\text { Desplazamiento al } \\
\text { colegio } \\
\text { Contexto de juego }\end{array}$} & Camina al colegio & 93 & $\begin{array}{c}0,80 \\
0,66-0,93\end{array}$ & $\begin{array}{c}0,61 \\
0,44-0,73\end{array}$ & --- & --- \\
\hline & Dentro de la casa & 103 & $\begin{array}{c}0,36 \\
0,13-0,60\end{array}$ & $\begin{array}{c}0,30 \\
0,11-0,47\end{array}$ & $\begin{array}{c}0,26 \\
0,1-0,45\end{array}$ & $\begin{array}{c}0,43 \\
0,26-0,57\end{array}$ \\
\hline & Fuera de la casa & 94 & $\begin{array}{c}0,35 \\
0,13-0,57\end{array}$ & $\begin{array}{c}0,53 \\
0,36-0,67\end{array}$ & $\begin{array}{c}0,25 \\
0,04-0,43\end{array}$ & $\begin{array}{c}0,31 \\
0,11-0,48\end{array}$ \\
\hline Práctica deportiva & Deporte organizado & 108 & $\begin{array}{c}0,72 \\
0,59-0,85\end{array}$ & $\begin{array}{c}0,81 \\
0,69-0,89\end{array}$ & $\begin{array}{c}0,57 \\
0,28-0,77\end{array}$ & $\begin{array}{c}0,77 \\
0,63-0,86\end{array}$ \\
\hline \multirow[t]{8}{*}{ Actividades de juego } & Camina & 101 & $\begin{array}{c}0,29 \\
0,10-0,50\end{array}$ & $\begin{array}{c}0,62 \\
0,47-0,73\end{array}$ & $\begin{array}{c}0,62 \\
0,47-0,73\end{array}$ & $\begin{array}{c}0,65 \\
0,52-0,75\end{array}$ \\
\hline & Baila & 100 & $\begin{array}{c}0,42 \\
0,23-0,61\end{array}$ & $\begin{array}{c}0,17 \\
-0,14-0,45\end{array}$ & $\begin{array}{c}0,83 \\
0,68-0,91\end{array}$ & $\begin{array}{c}0,46 \\
0,21-0,65\end{array}$ \\
\hline & Juega con balón & 102 & $\begin{array}{c}0,43 \\
0,25-0,61\end{array}$ & $\begin{array}{c}0,28 \\
0,05-0,48\end{array}$ & $\begin{array}{c}0,06 \\
-0,16-0,29\end{array}$ & $\begin{array}{c}0,40 \\
0,21-0,56\end{array}$ \\
\hline & Monta en bicicleta/patines & 101 & $\begin{array}{c}0,63 \\
0,48-0,78\end{array}$ & $\begin{array}{c}0,55 \\
0,29-0,74\end{array}$ & $\begin{array}{c}-0,04 \\
-0,31-0,24\end{array}$ & $\begin{array}{c}0,36 \\
0,11-0,53\end{array}$ \\
\hline & Salta lazo/trampolín & 97 & $\begin{array}{c}0,40 \\
0,20-0,61\end{array}$ & $\begin{array}{c}0,27 \\
-0,12-0,58\end{array}$ & $\begin{array}{c}-0,31 \\
-0,61-0,05\end{array}$ & $\begin{array}{c}0,41 \\
0,13-0,64\end{array}$ \\
\hline & Juega/nada en piscina & 99 & $\begin{array}{c}0,31 \\
0,12-0,51\end{array}$ & --- & $\begin{array}{c}0,47 \\
0,19-0,67\end{array}$ & $\begin{array}{c}0,60 \\
0,39-0,76\end{array}$ \\
\hline & Juega en el parque & 103 & $\begin{array}{c}0,42 \\
0,24-0,59\end{array}$ & $\begin{array}{c}0,09 \\
-0,24-0,41\end{array}$ & $\begin{array}{c}0,64 \\
0,46-0,76\end{array}$ & $\begin{array}{c}0,07 \\
-0,16-0,29\end{array}$ \\
\hline & Juega con amigos/vecinos & 108 & $\begin{array}{c}0,48 \\
0,29-0,66\end{array}$ & $\begin{array}{c}0,28 \\
0,06-0,47\end{array}$ & $\begin{array}{c}0,43 \\
0,25-0,59\end{array}$ & $\begin{array}{c}0,37 \\
0,19-0,53\end{array}$ \\
\hline
\end{tabular}

a Kappa: coeficiente kappa de Cohen, ${ }^{\mathrm{b}} \mathrm{IC}_{95 \%}$ : intervalo de confianza del $95 \%$, ${ }^{\mathrm{c}} \mathrm{CCl}$ : coeficiente de correlación intraclase, ${ }^{\mathrm{d}} \mathrm{ES}$ : entre semana, e $\mathrm{FS}$ : fin de semana

Cuadro 2. Reproducibilidad prueba-reprueba para los comportamientos sedentarios

\begin{tabular}{|c|c|c|c|c|c|c|}
\hline \multirow[t]{2}{*}{ Atributo } & \multirow[t]{2}{*}{ Ítem } & \multirow[t]{2}{*}{$\mathbf{N}$} & \multirow{2}{*}{$\begin{array}{c}{ }^{\text {a }} \text { Kappa }^{\mathrm{b}}\left(\mathrm{IC}_{95 \%}\right) \\
\text { Sí/No }\end{array}$} & \multicolumn{3}{|c|}{${ }^{\mathrm{c}} \mathbf{C C l}{ }^{\mathrm{b}} \mathrm{IC}_{95 \%}$} \\
\hline & & & & ${ }^{d}$ ES & ${ }^{\mathrm{e}} \mathbf{F S}$ & Total \\
\hline \multirow{2}{*}{$\begin{array}{l}\text { Desplazamiento al } \\
\text { colegio } \\
\text { Lectura }\end{array}$} & $\begin{array}{l}\text { Usa vehículo motorizado } \\
\text { para ir al colegio }\end{array}$ & 87 & $\begin{array}{c}0,84 \\
0,7-0,97\end{array}$ & $\begin{array}{c}0,79 \\
0,71-0,85\end{array}$ & ---- & ---- \\
\hline & El niño lee o la mamá le lee & 106 & $\begin{array}{c}0,65 \\
0,48-0,81\end{array}$ & $\begin{array}{c}0,41 \\
0,25-0,55\end{array}$ & $\begin{array}{c}0,54 \\
0,38-0,66\end{array}$ & $\begin{array}{c}0,37 \\
0,21-0,52\end{array}$ \\
\hline \multirow[t]{3}{*}{ Actividades en pantalla } & Ver televisión & 107 & $\begin{array}{c}0,49 \\
-0,11-1,0\end{array}$ & $\begin{array}{c}0,49 \\
0,34-0,61\end{array}$ & $\begin{array}{c}0,39 \\
0,23-0,53\end{array}$ & $\begin{array}{c}0,52 \\
0,38-0,64\end{array}$ \\
\hline & Uso de computador & 107 & $\begin{array}{c}0,71 \\
0.57-0,85\end{array}$ & $\begin{array}{c}0,66 \\
0,54-0,75\end{array}$ & $\begin{array}{c}0,65 \\
0,52-0,75\end{array}$ & $\begin{array}{c}0,70 \\
0,59-0,78\end{array}$ \\
\hline & Uso de videojuegos & 104 & $\begin{array}{c}0,62 \\
0,46-0,78\end{array}$ & $\begin{array}{c}0,39 \\
0,22-0,54\end{array}$ & $\begin{array}{c}0,24 \\
0,1-0,41\end{array}$ & $\begin{array}{c}0,32 \\
0,15-0,47\end{array}$ \\
\hline \multirow[t]{2}{*}{ Tiempo de sueño } & Siesta en el día & 97 & $\begin{array}{c}0,57 \\
0,40-0,74\end{array}$ & $\begin{array}{c}0,60 \\
0,47-0,71\end{array}$ & $\begin{array}{c}0,85 \\
0,78-0,90\end{array}$ & $\begin{array}{c}0,62 \\
0,49-0,72\end{array}$ \\
\hline & Dormir de noche & 118 & ---- & $\begin{array}{c}0,70 \\
0,60-0,78\end{array}$ & $\begin{array}{c}0,40 \\
0,24-0,55\end{array}$ & $\begin{array}{c}0,57 \\
0,44-0,68\end{array}$ \\
\hline Cursos extracurriculares & Actividades en sedente & 104 & $\begin{array}{c}0,46 \\
0,26-0,67\end{array}$ & $\begin{array}{c}0,13 \\
-0,1-0,31\end{array}$ & $\begin{array}{c}0,64 \\
0,52-0,74\end{array}$ & $\begin{array}{c}0,36 \\
0,19-0,51\end{array}$ \\
\hline
\end{tabular}

${ }^{a}$ kappa: coeficiente kappa de Cohen, ${ }^{b} \mathrm{IC}_{95 \%}$ : intervalo de confianza del $95 \%$,

${ }^{\mathrm{C}} \mathrm{CCl}$ : coeficiente de correlación intraclase, ${ }^{\mathrm{d}} \mathrm{ES}$ : entre semana, ${ }^{\mathrm{e}} \mathrm{FS}$ : fin de semana

actividades usuales, la semana anterior), el tiempo entre las dos mediciones para los estudios de reproducibilidad y el número de ítems para los estudios de consistencia interna, entre otros.
Al comparar la definición del constructo de actividad física de este estudio con la de otros cuestionarios, queda clara la gran variación de tales definiciones, pues se indaga por el tiempo (en minutos) dedicado 
a cada nivel de intensidad de la actividad física (12), por las preferencias (13), por el tiempo de juego al aire libre $(14,16)$, por la atracción por la actividad física (17), por las preferencias de los niños (21) y por las percepciones de los padres (22), lo cual marca una diferencia en la medición de base y en la aproximación para establecer el nivel de actividad física de los niños, así como el tiempo invertido en comportamientos sedentarios, con el fin de establecer el cumplimiento de las recomendaciones internacionales vigentes (8-11).

El nivel de consistencia interna de la información sobre la actividad física, fue moderado y osciló entre 0,59 y 0,64 , y el correspondiente a los comportamientos sedentarios fue bajo, entre 0,22 y 0,34 . Es importante explicar cuál es el fondo conceptual de lo que se evalúa con el coeficiente alfa de Cronbach, con el cual se responde a la pregunta: ¿Todos los ítems de la escala pertenecen al mismo constructo? En este caso los constructos fueron dos: actividad física y comportamientos sedentarios; cabe señalar, además, que este coeficiente corresponde a la correlación interna de los puntajes obtenidos y no de la escala en sí misma, y que solo tiene significado si la escala es unidimensional, es decir, si los ítems que contiene representan un solo constructo, lo cual solo puede demostrarse mediante el modelo de Rasch, el cual hasta ahora se ha utilizado poco para el análisis de cuestionarios sobre actividad física en adultos (28). Por otra parte, este coeficiente depende del número de ítems, lo que implica que, en la medida en que este aumenta, aumenta también la consistencia interna (24).

La consistencia interna se evaluó sólo en tres de los cuestionarios revisados, con resultados similares para el tiempo dedicado a la actividad física, con 12 ítems en el caso de Harro (alfa=0,69) (12), para la atracción por la actividad física establecida mediante una entrevista a los niños, con 25 ítems (alfa=0,55-0,74) (17), y para el cuestionario sobre el ambiente familiar y los factores del comportamiento relacionados con el sobrepeso y la obesidad, con 21 ítems (alfa $=0,72$ ) (18). Estos datos evidencian que los resultados obtenidos para la actividad física, fueron similares

Cuadro 3. Promedio de las diferencias y límites de acuerdo del $95 \%$ para el tiempo en minutos diarios dedicados a diferentes actividades de juego

\begin{tabular}{lllcc}
\hline Atributo & Ítem & \multicolumn{1}{c}{${ }^{a}$ ES } & ${ }^{\text {b FS }}$ & Total \\
\hline Desplazamiento al colegio & Camina al colegio & 14,$5 ;-0,9-16,3$ & ---- & --- \\
Contexto de juego & Dentro de la casa & 95,$6 ;-5,5-106,6$ & 180,$3 ;-11,0-202,2$ & 91,$2 ;-7,4-106,0$ \\
& Fuera de la casa & 53,$5 ;-0,6-54,7$ & 119,$8 ;-16,2-152,3$ & 68,$4 ;-1,1-70,6$ \\
Práctica deportiva & Deporte organizado & 37,$0 ; 1,4-34,3$ & 65,$3 ; 5,9-53,5$ & 38,$2 ; 2,4-33,3$ \\
Actividades de juego & Camina & 22,$6 ;-1,7-25,9$ & 57,$9 ;-6,1-70,1$ & 28,$9 ;-1,1-31,0$ \\
& Baila & 16,$9 ; 0,0-16,8$ & 45,$5 ;-0,6-46,7$ & 20,$6 ;-0,1-20,7$ \\
& Juega con balón & 33,$8 ;-0,8-35,4$ & 68,$9 ;-1,7-72,4$ & 32,$9 ;-0,9-34,7$ \\
& Monta en bicicleta/patines & 32,$0 ; 1,9-28,2$ & 90,$9 ; 3,3-84,4$ & 39,$2 ; 2,6-33,8$ \\
& Salta lazo/trampolín & 12,$3 ;-1,1-14,4$ & 44,$4 ; 0,16-44,1$ & 16,$3 ; 0,1-16,2$ \\
& Juega/nada en piscina & 15,$9 ;-2,5-20,8$ & 91,$9 ;-6,8-78,2$ & 27,$7 ; 0,9-25,8$ \\
& Juega en el parque & 23,$7 ;-0,26-24,3$ & 64,$8 ;-0,3-65,3$ & 31,$5 ;-2,2-35,9$ \\
& Juega con amigos/vecinos & 46,$9 ;-9,3-65,5$ & 106,$0 ;-4,0-114,0$ & 48,$3 ;-7,1-62,5$ \\
\hline
\end{tabular}

a ES: entre semana, ${ }^{\text {b }} \mathrm{FS}$ : fin de semana

Cuadro 4. Promedio de las diferencias y límites de acuerdo del $95 \%$ para el tiempo en minutos diarios dedicado a actividades sedentarias

\begin{tabular}{llccc}
\hline Atributo & Ítem & ${ }^{a}$ ES & ${ }^{b}$ FS & Total \\
\hline Desplazamiento al colegio & Usa vehículo motorizado para & 23,$2 ;-0,31-25,8$ & ---- & --- \\
& ir al colegio & & & \\
Lectura & El niño lee o la mamá le lee & 50,$8 ; 2,8-45,1$ & 44,$0 ; 0,9-42,1$ & 45,$9 ; 2,1-41,7$ \\
Actividades en pantalla & Ver televisión & 111,$7 ; 5,9-99,9$ & 220,$0 ; 17,4-185,3$ & 121,$0 ; 7,1-106,8$ \\
& Uso de computador & 47,$2 ; 4,1-38,9$ & 89,$6 ; 10,1-69,4$ & 46,$9 ; 4,2-38,5$ \\
& Uso de videojuegos & 51,$4 ;-2,0-55,5$ & 129,$1 ; 5,4-118,3$ & 62,$9 ; 0,4-62,0$ \\
Tiempo de sueño & Siesta en el día & 50,$7 ;-2,6-56,0$ & 25,$1 ; 2,5-20,1$ & 41,$6 ;-1,3-44,1$ \\
& Dormir de noche & 83,$0 ;-5,0-93,0$ & 158,$5 ;-1,6-161,8$ & 99,$1 ;-3,6-107,2$ \\
Cursos extracurriculares & Actividades en sedente & 47,$1 ;-0,2-47,2$ & 54,$8 ;-2,6-60,0$ & 39,$6 ;-0,9-41,4$ \\
\hline
\end{tabular}

${ }^{a}$ ES: entre semana, ${ }^{\text {b }} \mathrm{FS}$ : fin de semana 
a los encontrados previamente. En cuanto a los comportamientos sedentarios, es probable que se deba discriminar mejor el tipo de comportamiento y que con un número mayor de ítems se obtengan resultados similares a los encontrados para la actividad física.

En este caso, tanto el coeficiente kappa de Cohen como el coeficiente de correlación intraclase se emplearon para responder a la siguiente pregunta: ¿ se obtienen los mismos resultados al repetirse la prueba? (24). En este contexto, además del período de recuerdo evocado, hay una variable importante que debe considerarse y es el tiempo de medición entre las dos aplicaciones. En este estudio el cuestionario elaborado evoca la semana anterior, en tanto que el tiempo entre las dos aplicaciones fue de una semana.

Los resultados de reproducibilidad para la práctica de actividad física (sí o no) fueron buenos en los ítems de desplazamiento al colegio y la práctica de un deporte organizado, y entre moderados y sustanciales en los demás (cuadro 1); entre los comportamientos sedentarios, el transporte motorizado al colegio, la lectura y el uso de computador y de consolas, también arrojaron valores de kappa buenos, mientras que los ítems restantes se ubicaron en la categoría de sustancial (cuadro 2). Una posible explicación de estos resultados es la facilidad con que los padres recuerdan rutinas establecidas, como acompañar al niño al colegio y a las prácticas de deportes organizados; por otra parte, es posible que las reglas para el uso de computadores y consolas de videojuegos en el hogar contribuyan al resultado señalado.

En lo concerniente al tiempo invertido en cada actividad, se encontraron mejores coeficientes para el período entre semana que para el fin de semana, con excepción del juego en la piscina, en el parque y con los amigos, posiblemente por las mismas razones ya señaladas, y porque durante el fin de semana los comportamientos y las reglas paternas pueden ser más abiertas y se ejerce un menor control. Es posible, también, que por tratarse de actividades de juego libre haya una mayor variabilidad en el comportamiento de los niños en sí mismo, y el resultado no obedezca a problemas del cuestionario, aspecto sobre el que ya se ha llamado la atención en cuestionarios dirigidos a padres (20).

La comparación de los resultados de este estudio con los de otras publicaciones, demuestra que fueron inferiores a los de Telford, et al. (15), quienes evaluaron el tipo, la frecuencia y la duración de la actividad física vigorosa y la actividad física de moderada a vigorosa en un día de semana y en uno típico de fin de semana con un intervalo de 14 días, y se obtuvieron coeficientes de correlación intraclase entre 0,76 y 0,87 . Una situación similar se presenta con los datos de Adami, et al. (19), en cuyo estudio se indagó sobre la actividad física y los comportamientos sedentarios del día anterior con un período de tres días entre las dos mediciones, y se obtuvieron coeficientes de correlación intraclase entre 0,87 y 0,97.

Se encontraron coeficientes similares a los de este estudio en los resultados de Dwyer, et al. (20), con coeficientes de correlación intraclase entre 0,44 y 0,64 para los comportamientos sedentarios y la actividad física ligera, moderada y vigorosa durante el día anterior entre semana y en el último fin de semana, con un intervalo de 14 días entre las dos mediciones; así como en el trabajo de Veitch, et al. (16), en el cual dicho coeficiente se ubicó entre 0,40 y 0,82 entre semana y 0,18 y 0,77 durante el fin de semana en el curso de una semana típica y con un período de 14 días entre las dos mediciones.

Al analizar el promedio de las diferencias entre las dos mediciones en cuanto a los minutos diarios dedicados a cada tipo de actividad, así como de los límites de acuerdo del $95 \%$, se encontraron, en general, promedios cercanos a cero e inferiores para las actividades entre semana comparadas con las de fin de semana. En cuanto a los límites superior e inferior, los rangos estuvieron entre 30 y 50 minutos para la mayoría de los ítems; cabe señalar que para el juego, ver televisión y dormir en la noche, los rangos fueron muy amplios, lo que podría deberse a una mayor variabilidad en el comportamiento de los niños o a una menor calidad en el registro por parte de los padres, ya que son actividades a las que no se presta mayor atención por ser comunes o de tipo familiar.

Solo el trabajo de Adami, et al., permite comparaciones con esta prueba, en la que se registraron promedios de diferencias entre 1 y 5 minutos, con límites superiores e inferiores entre 40 y 60 minutos, datos similares a los del presente estudio para la mayoría de actividades, tanto las relacionadas con la actividad física como con los comportamientos sedentarios.

Entre las limitaciones del estudio es importante señalar la posible sobrestimación de las actividades y los tiempos dedicados a la actividad física, así como la subestimación de estos mismos atributos en el caso de los comportamientos 
sedentarios, derivadas de la naturaleza subjetiva de los cuestionarios, lo cual ya se ha documentado previamente (2-5). Por otro lado, es necesario tener en cuenta la naturaleza cambiante y compleja de la actividad física que, en el caso de los menores de 10 años, está directamente relacionada con el juego y se ve condicionada por múltiples factores determinantes, tanto biológicos, como sociales y de contexto $(6,7)$.

A pesar de las limitaciones identificadas, este estudio tiene fortalezas, entre las que se puede resaltar el hecho de que la escogencia de las actividades y el número de ítems contemplados en el cuestionario se basó en la caracterización hecha en Bucaramanga y su área metropolitana en el primer semestre del 2012 (datos sin publicar), y en la selección aleatoria de colegios pertenecientes a todos los niveles socioeconómicos. En cuanto al diseño del cuestionario, la definición de actividad física y comportamientos sedentarios se diferenció de la de otros instrumentos y, además, se indagó por las actividades entre semana y en fin de semana.

Este es el primer cuestionario diseñado y evaluado en el ámbito latinoamericano que resulta comparable con otros similares diseñados en Australia $(15-17,20)$ y en Estados Unidos $(14,18)$, con un nivel de confiabilidad aceptable para utilizarlo en un contexto de estudios de población (con todas las ventajas y limitaciones que estos implican), estimar el nivel de actividad física y comportamiento sedentario en niños de este grupo etario y contribuir así a describir las tendencias en el tiempo, así como proponer, desarrollar y evaluar políticas de salud pública dirigidas a mejorar la salud de los menores de 10 años.

El cuestionario, asimismo, podría aplicarse en otros países latinoamericanos ampliando la evaluación de sus propiedades psicométricas, con lo que se dispondría de una herramienta para comparar la situación de la actividad física y el comportamiento sedentario y, además, seguir y evaluar las diferentes intervenciones propuestas para incrementar el estilo de vida saludable de la población desde la primera infancia.

\section{Conflicto de intereses}

Los autores declaran no tener conflicto de intereses.

\section{Financiación}

Los autores agradecen a la Universidad Industrial de Santander por la financiación concedida para la realización de este proyecto.

\section{Referencias}

1. Organización Mundial de la Salud. Recomendaciones mundiales sobre actividad física para la salud. 2010. Fecha de consulta: 15 de abril de 2014. Disponible en: http:// whqlibdoc.who.int/publications/2010/9789243599977_spa.pdf.

2. Trost SG. Measurement of physical activity in children and adolescents. Am J Lifestyle Med. 2007;1:299-314. http:// dx.doi.org/10.1177/1559827607301686

3. Pate RR, O'Neill JR, Mitchell J. Measurement of physical activity in preschool children. Med Sci Sports Exerc. 2010;42:508-12. http://dx.doi.org/10.1249/MSS. Ob013e3181cea116

4. Lewicka M, Farrell L. Physical activity measurement in children 2-5 years of age. 2007. Fecha de consulta: 2 de mayo de 2014. Disponible en: http://sydney.edu.au/ medicine/public-health/cpah/pdfs/2007_pa measurement farrell.pdf

5. Loprinzi PD, Cardinal BJ. Measuring children's physical activity and sedentary behaviors. J Exerc Sci Fit. 2011;9:1523. http://dx.doi.org/10.1016/S1728-869X(11)60002-6

6. Dwyer G, Baur L, Hardy L. The challenge of understanding and assessing physical activity in preschool-age children: Thinking beyond the framework of intensity, duration and frequency of activity. J Sci Med Sport. 2008;12:534-6. http:// dx.doi.org/10.1016/j.jsams.2008.10.005

7. Burdette HL, Whitaker RC. Resurrecting free play in young children: Looking beyond fitness and fatness to attention, affiliation and affect. Arch Pediatr Adolesc Med. 2005;159:4650. http://dx.doi.org/10.1001/archpedi.159.1.46

8. National Association for Sport and Physical Education. Active Start. A statement of physical activity guidelines for children from birth to age 5. 2nd edition. Sewickley, PA: AAHPERD Publications Fulfillment Center; 2009.

9. Tremblay MS, Leblanc AG, Carson V, Choquette L, Gorber SC, Dillman C, et al. Canadian Physical Activity Guidelines for the Early Years (aged 0-4 years). Appl Physiol Nutr Metab. 2012;37:345-56. http://dx.doi.org/10.1139/ h2012-018

10. Australian Government Department of Health and Ageing. Get up \& grow: Healthy eating and physical activity for early childhood - Staff and Carer Book. Fecha de consulta: 18 de febrero de 2015. Disponible en: http:// www.health.gov.au/internet/main/publishing.nsf/Content/2C DB3A000FE57A4ECA257BF0001916EC/\$File/HEPA\%20 -\%20B5\%20Book\%20-\%20Staff\%20and\%20Carer\%20 Book LR.pdf

11. Tremblay MS, Leblanc AG, Carson V, Choquette L, Gorber SC, Dillman C, et al. Canadian Sedentary Behavior Guidelines for the Early Years (aged 0-4 years). Appl Physiol Nutr Metab. 2012;37:370-91. http://dx.doi.org/10.1139/ h2012-019

12. Harro M. Validation of a questionnaire to assess physical activity of children ages 4-8 years. Res Q Exerc Sport. 1997;68:259-68. http://dx.doi.org/10.1080/02701367.1997. 10608007

13. Sekine $M$, Yamagami T, Chen $\mathbf{X}$, Hayashikawa $Y$, Hamanishi S, Kagamimori S. Validity of a questionnaire evaluating physical activity level in young children. Environ Health Prev Med. 2002;6:264-7. http://dx.doi.org/10.1007/ BF02897980 
14. BurdetteH, Whitaker R, Daniels S. Parental report of outdoor playtime as a measure of physical activity in preschool-aged children. Arch Pediatr Adolesc Med. 2004:158;353-7. http:// dx.doi.org/10.1001/archpedi.158.4.353

15. Telford A, Salmon J, Jolley D, Crawford D. Reliability and validity of physical activity questionnaires for children: The Children`s Leisure Activities Study Survey (CLASS). Pediatr Exerc Sci. 2004;16:64-78.

16. Veitch J, Salmon J, Ball K. The validity and reliability of an instrument to assess children's outdoor play in various locations. J Sci Med Sport. 2009;12:579-82. http://dx.doi. org/10.1016/j.jsams.2008.09.001

17. Rose E, Larkin D, Hands B, Howard B, Parker H. Evidence for the validity of the Children's Attraction to Physical Activity questionnaire (CAPA) with young children. J Sci Med Sport. 2009;12:573-8. http://dx.doi.org/10.1016/j. jsams.2009.05.009

18. Ihmels M, Welk G, Eisenmann J, Nusser S. Development and preliminary validation of a Family Nutrition and Physical Activity (FNPA) screening tool. Int $\mathrm{J}$ Behav Nutr Phys Act. 2009;6:14. http://dx.doi.org/10.1186/1479-5868-6-14

19. Adami F, Cruciani F, Douek M, Sewell CD, Mariath AB, de Fragas-Hinnig $\mathbf{P}$, et al. Reliability of the Brazilian version of the physical activity checklist interview in children. Rev Saúde Pública. 2011;45:321-33. http://dx.doi.org/10.1590/ S0034-89102011000200011

20. Dwyer G, Hardy L, Peat J, Baur L. The validity and reliability of a home environment preschool-age physical activity questionnaire (Pre-PAQ). Int J Behav Nutr Phys Act. 2011;8:86. http://dx.doi.org/10.1186/1479-5868-8-86
21. Bielemann R, Reichert F, Paniz V, Gigante D. Validation of the Netherlands physical activity questionnaire in Brazilian children. Int J Behav Nutr Phys Act. 2011;8:45. http://dx.doi. org/10.1186/1479-5868-8-45.

22. Bacardi-Gascón M, Reveles-Rojas C, Woodward-López G, Crawford P, Jiménez-Cruz A. Validity of a physical activity questionnaire used with parents of preschool children in México. Nutr Hosp. 2011;26:244-5.

23. Camargo DM, Orozco LC. Factores asociados a uso de medios electrónicos en niños desde preescolar hasta cuarto. Biomédica. 2013;33:175-85. http://dx.doi.org/10.7705/ biomedica.v33i2.779

24. Orozco LC. Confiabilidad de la consistencia, reproducibilidad, acuerdo y algo más. En: Orozco-Vargas LC, editor. Medición en salud. Diagnóstico y evaluación de resultados: un manual critico más allá de lo básico. Bucaramanga: Publicaciones UIS; 2010. p. 73-103.

25. Landis JR, Koch GG. The measurement of observer agreement for categorical data. Biometrics. 1997;33:159-74.

26. Bland JM, Altman DG. Statistical methods for assessing agreement between two methods of clinical measurement. Lancet. 1986;i:307-10.

27. StataCorp. Stata: Release 12. Statistical Software. College Station, TX: StataCorp LP; 2011.

28. Hopman-Rock M, Dusseldorp E, Chorus A, Jacobusse G, Ruetten A, van Buuren S. Response conversion for improving comparability of international physical activity data. J Phys Act Health. 2012;9:29-38. 


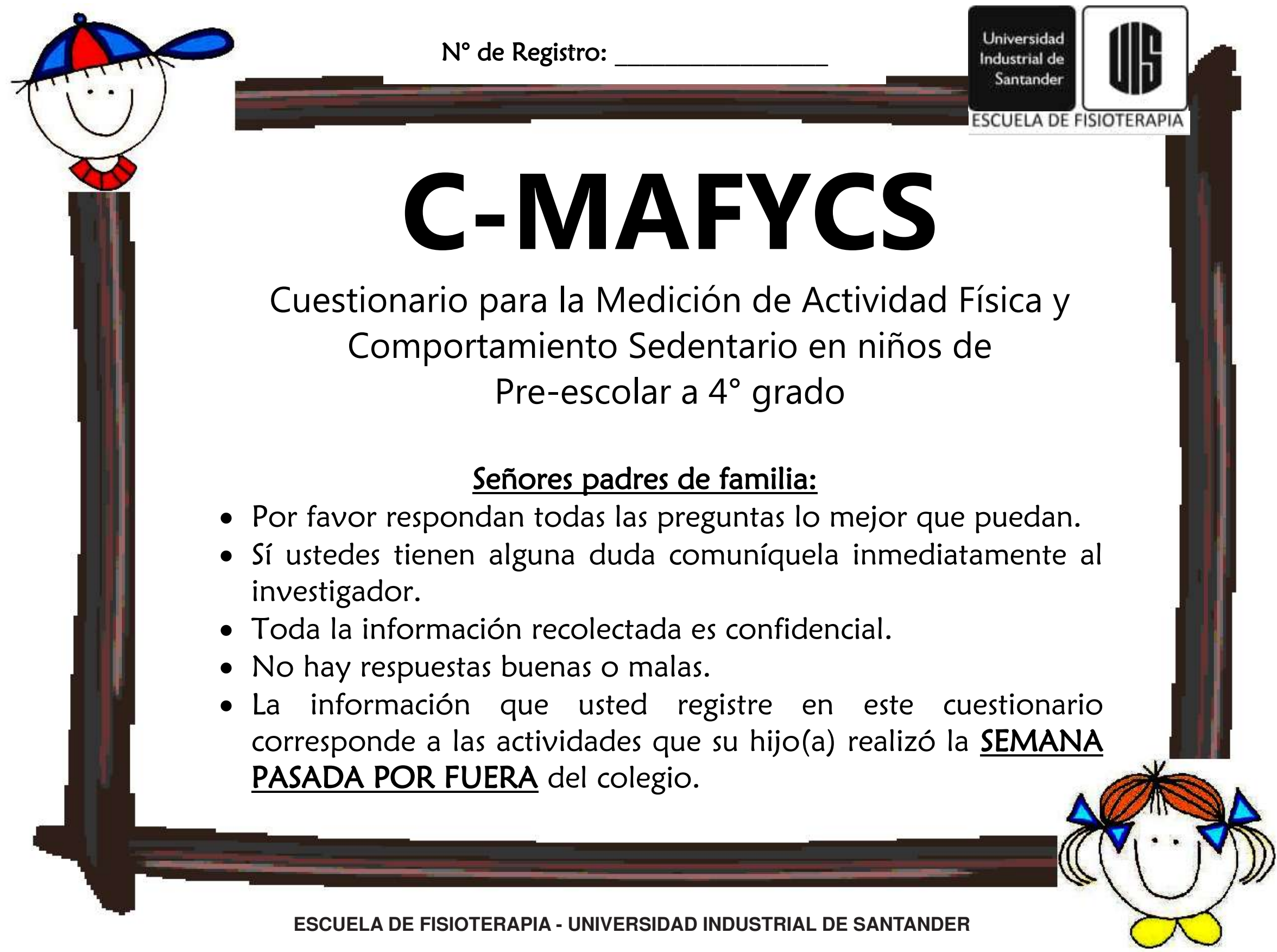


FECHA: DÍA MES [ ] $A \tilde{N} O$

1. INSTITUCIÓN EDUCATIVA:

¿Quién responde la encuesta?

\section{[}

Madre

]

\section{INFORMACIÓN DEL ACUDIENTE}

NOMBRES Y APELLIDOS:

EDAD: años cumplidos

GÉNERO: Masculino Femenino

NIVEL DE ESCOLARIDAD:

¿Cuántos años de primaria terminó? años

¿Cuántos años de educación técnica terminó? ¿TRABAJA FUERA DE LA CASA?

Si años

¿Cuántos años de bachillerato terminó? años No $\square$

¿Cuántos años de universidad terminó? años

Sí respondió Sí a la pregunta anterior, por favor responda cuánto tiempo:

Menos de medio tiempo $\square$ Medio Tiempo $\square$ Tiempo Completo $\square$

INGRESOS ECONÓMICOS FAMILIARES MENSUALES EN SALARIO MÍNIMO LEGAL VIGENTE (SMLV) (\$566.700):

Menos de 1 SMLV $\square$ Entre 1 y 2 SMLV $\square$ Entre 3 y 4 SMLV $\square$ Más de 6 SMLV $\square$ Entre 5 y 6 SMLV

\section{INFORMACIÓN DEL NIÑO}

NOMBRES Y APELLIDOS:

EDAD: años cumplidos

FECHA DE NACIMIENTO: día [ JORNADA ESCOLAR: mes [ ] año [ HORA DE INGRESO AL COLEGIO:

GRADO ESCOLAR: Pre-jardín [_] Jardín [_] Transición [_] 


\section{ACTIVIDAD FÍSICA}

Actividades en las que necesita un mayor esfuerzo, aumentan los latidos del corazón, la respiración y a veces suda, por ejemplo: caminar, correr, jugar con la pelota o practicar algún deporte.
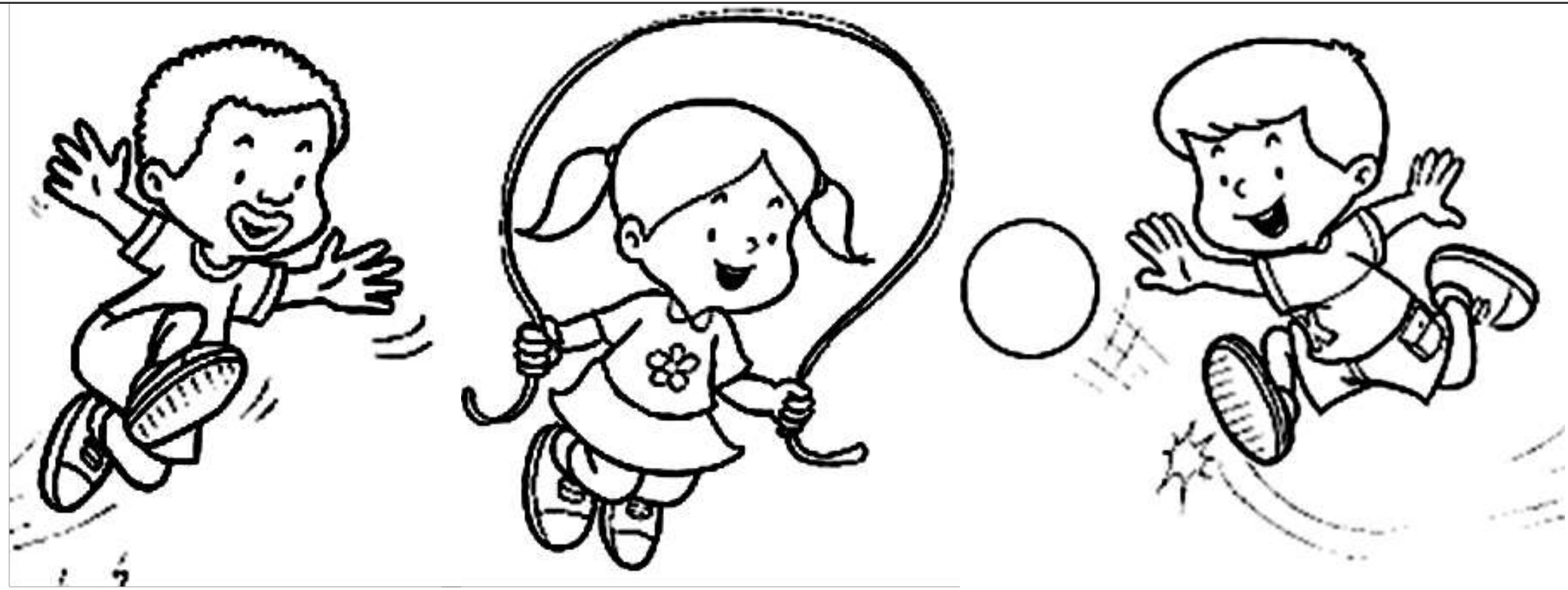

RECUERDE: La información que usted registre en este cuestionario corresponde a las actividades que su hijo realizó la SEMANA PASADA POR FUERA del colegio. 


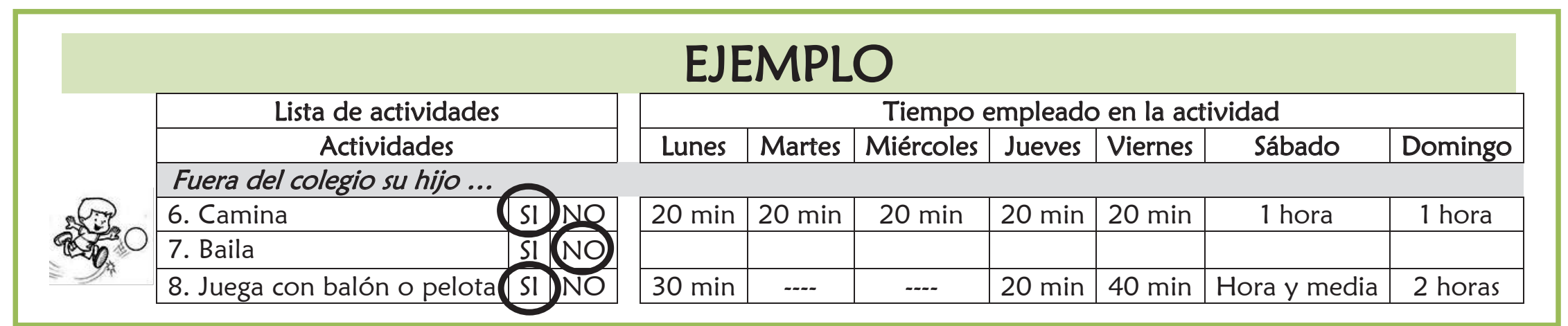

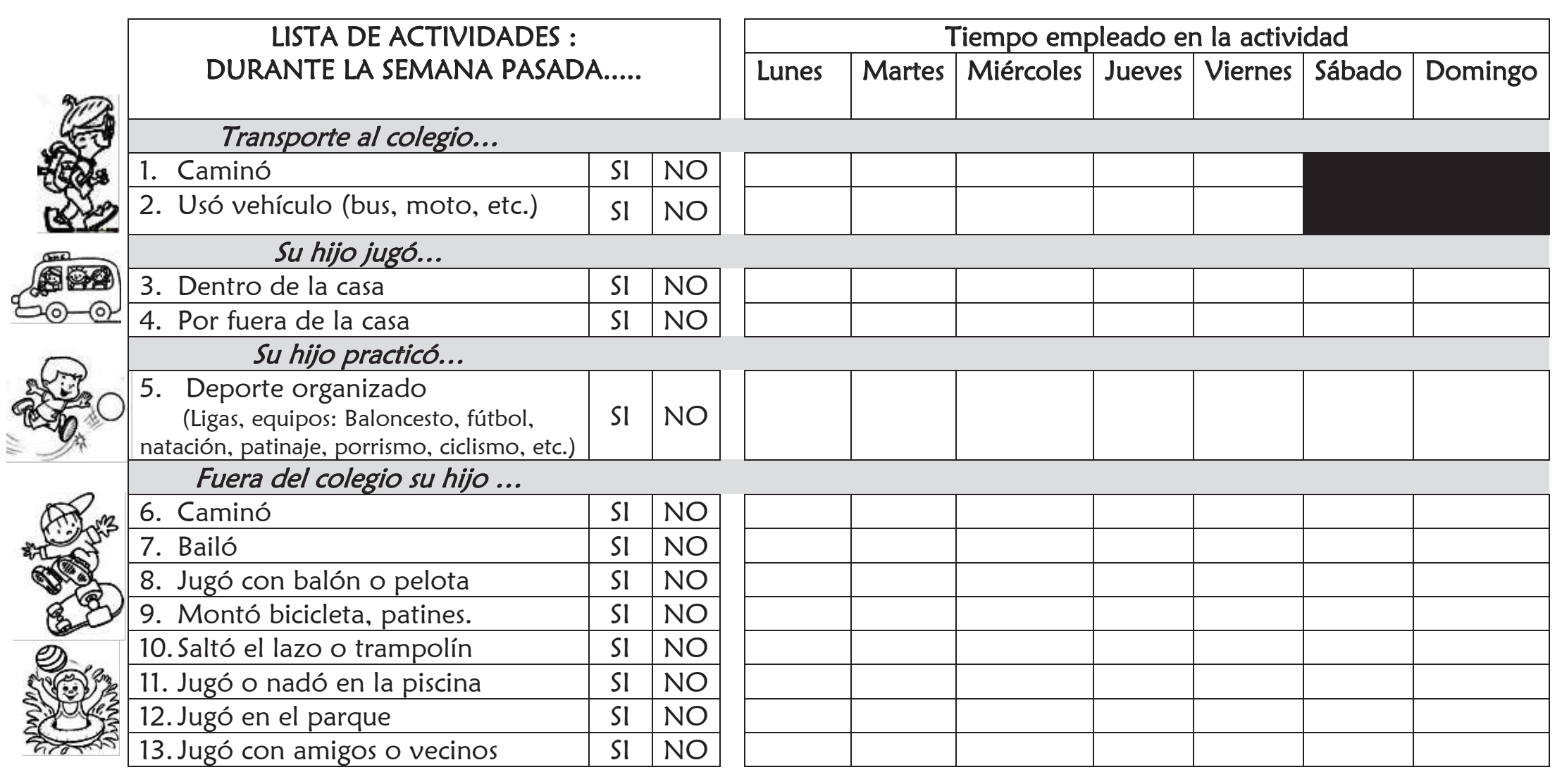




\section{COMPORTAMIENTOS cuck! SEDENTARIOS}

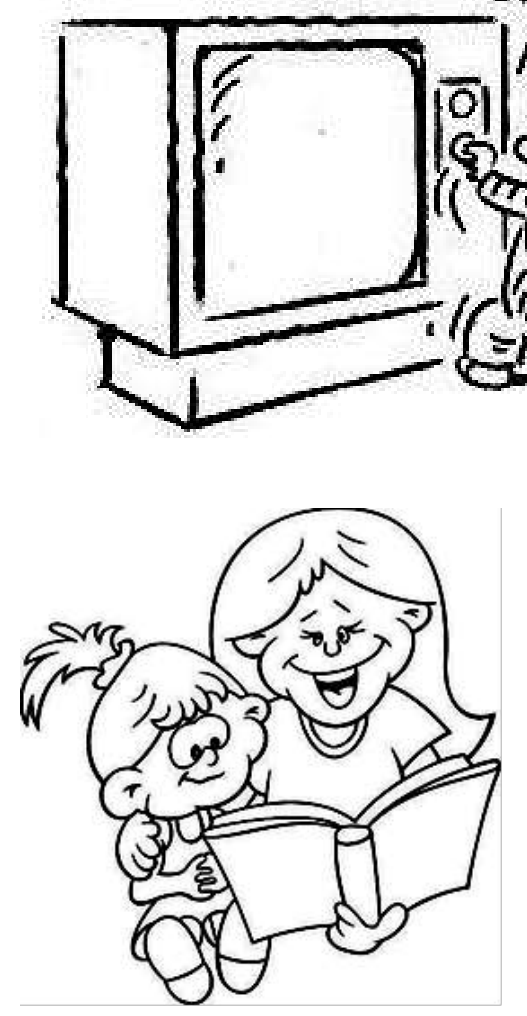

Requieren mínimo o ningún movimiento y necesita poco esfuerzo. Por ejemplo: ver televisión, leer un libro ó usar el computador, entre otras.

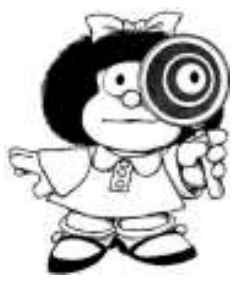

RECUERDE: La información que usted registre en este cuestionario corresponde a las actividades que su hijo realizó la SEMANA PASADA POR FUERA del colegio. 


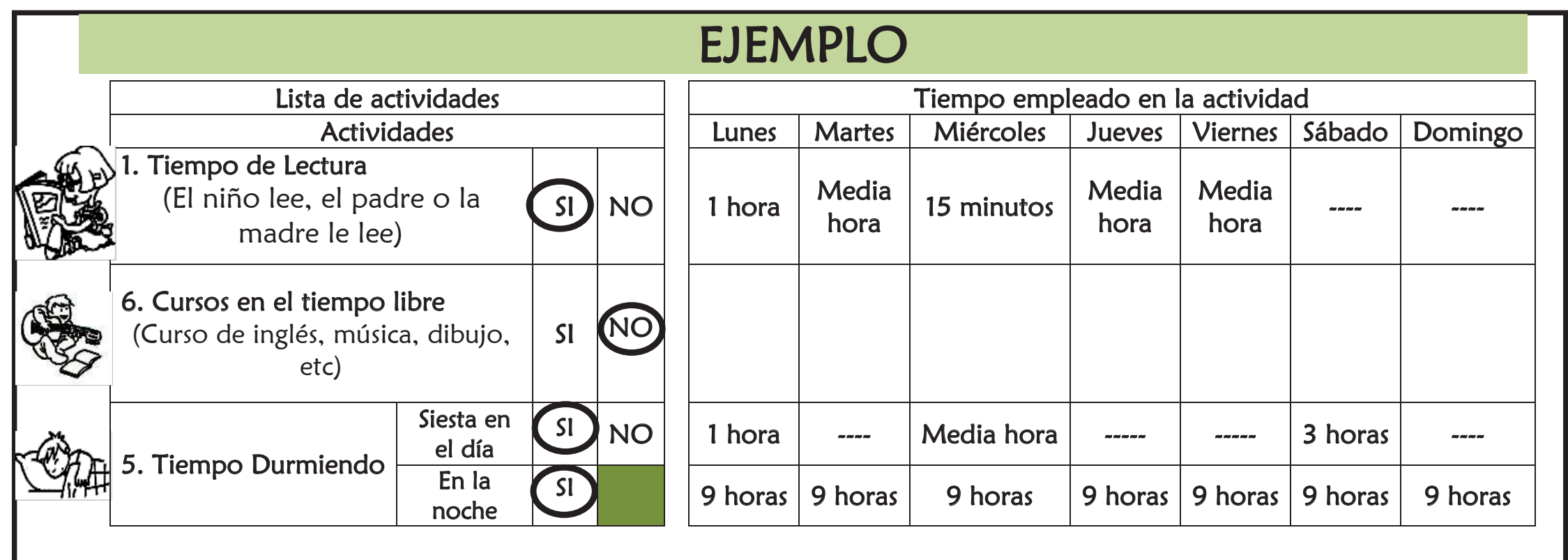

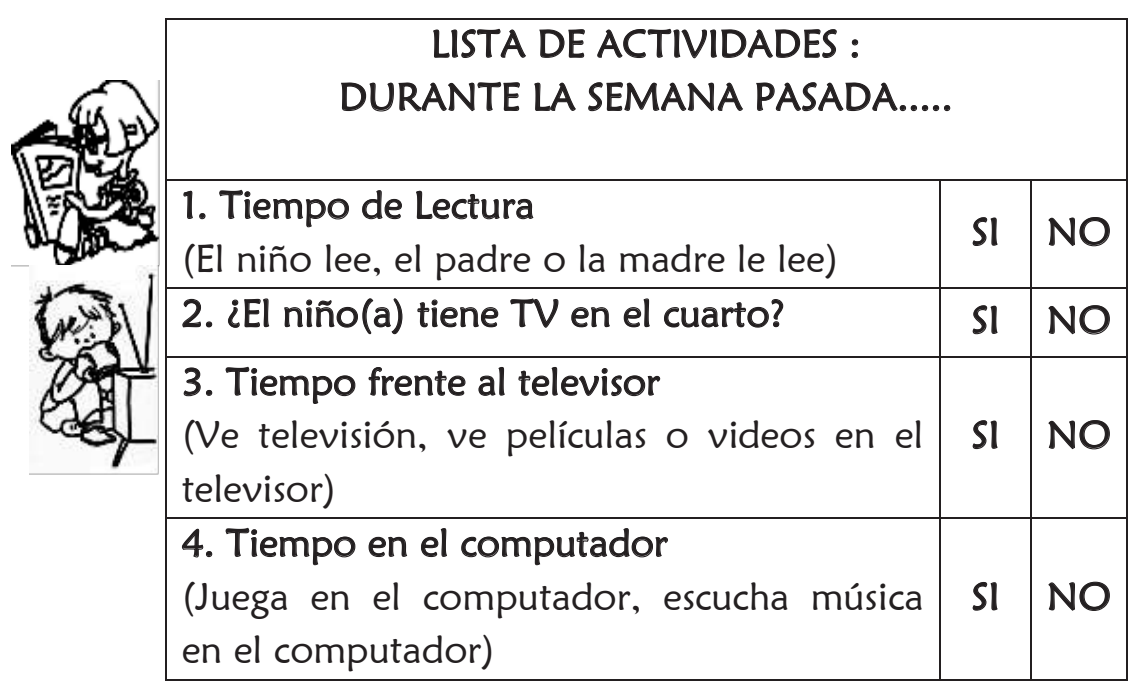

\begin{tabular}{|l|l|l|l|l|l|l|}
\hline \multicolumn{7}{|c|}{ Tiempo empleado en la actividad } \\
\hline Lunes & Martes & Miércoles & Jueves & Viernes & Sábado & Domingo \\
\hline & & & & & & \\
\hline & & & & & & \\
\hline & & & & & & \\
\hline & & & & & & \\
\hline
\end{tabular}




\begin{tabular}{|c|c|c|c|}
\hline DU & $\begin{array}{l}\text { ACTIVIDADES : } \\
\text { SEMANA PASADA.. }\end{array}$ & & \\
\hline $\begin{array}{l}\text { 5. Tiempo } \\
\text { (Juega con } \\
\text { Play-Station } \\
\text { manuales cc }\end{array}$ & $\begin{array}{l}\text { las } \\
\text { como X-Box, Wii, } \\
\text { con videojuegos } \\
\text { do DS, etc.) }\end{array}$ & SI & NO \\
\hline 6. Tiempo & $\begin{array}{l}\text { Siesta en el } \\
\text { día }\end{array}$ & SI & NO \\
\hline Durmiendo & En la noche & SI & \\
\hline $\begin{array}{l}\text { 7. Cursos c } \\
\text { en el tiempc }\end{array}$ & isica, dibujo, etc. & SI & NO \\
\hline
\end{tabular}

\section{INFORMACIÓN SOBRE LA JORNADA ESCOLAR}

El niño asiste al jardín o pre-escolar?

Si [

No [__ ]

Si la respuesta es afirmativa, por favor registre:

Hora de entrada:

Hora de salida:

GRADO ESCOLAR: Pre-jardín [_] Jardín [_] Transición [_] 